\title{
Avaliação de nitrogênio e fósforo dos efluentes tratados dos abatedouros na cidade de Pelotas/RS
}

\section{Assessment of nitrogen and phosphorus of treated effluents from slaughterohouses in the city of Pelotas/RS}

\author{
Daniel Lucas Costa Rodrigues ${ }^{1}$ Marcelo Peske Hartwig, Michel David Gerber
}

${ }^{1}$ Tecnologo em Saneamento Ambiental, Instituto Federal de Educação, Ciência e Tecnologia Sul-Rio-Grandense, Pelotas, RS, Brasil

\section{Resumo}

O setor agroindustrial cárneo é de grande importância econômica no Rio Grande do Sul, entretanto essa atividade também é reconhecida pelo potencial poluidor elevado. Considerando as dificuldades para o atendimento aos restritivos padrões de emissão de nitrogênio e fósforo, o presente trabalho teve por objetivo avaliar os efluentes tratados de quatro abatedouros da região de Pelotas/RS, relacionando-os aos padrões de emissão estabelecidos pela Resolução CONSEMA $n^{\circ}$ 128/2006, após quatro anos do final do prazo legal estabelecido. Todas as empresas avaliadas atendem ao padrão de lançamento estabelecido para fósforo, porém em relação ao parâmetro NTK, apenas $50 \%$ das empresas avaliadas atenderam ao preconizado na legislação, por concentração ou por eficiência mínima de remoção. Os resultados indicaram ainda que dois abatedouros precisam continuar investindo no sistema de tratamento de efluentes para atender a legislação ambiental vigente.

Palavras-chave: suínos, bovinos, NTK, P, legislação ambiental.

\begin{abstract}
The flesh agroindustrial sector is of great economic importance in Rio Grande do Sul, however this activity is also recognized by the high pollution potential. Considering the difficulties to fulfill the stringent nitrogen and phosphorus emission standards, this study aimed to assess the treated effluents of four slaughterhouses of the region of Pelotas/RS, relating them to the emission standards established by CONSEMA Resolution number 128/2006, after four years of the end of the period prescribed by law. All assessed companies fulfill the established discharge standard for phosphorus, but in relation to the KTN parameter, only 50\% of the assessed companies fulfilled what is recommended by law, by concentration or by minimum removal efficiency. The results also indicated that two slaughterhouses must continue investing in the effluents treatment system to fulfill the applicable environmental law.
\end{abstract}

Keywords: pigs, cattle, KTN, P, eviromental legislation. 


\section{Introdução}

A produção de alimentos é de fundamental importância tanto para a manutenção da vida humana (VIEIRA, 2013), como para o desenvolvimento de diversos países, pois influenciam nos aspectos sociais, políticos e econômicos (JAIME, 2011).

O Brasil é líder mundial de produção e exportação de alimentos, como a soja, o milho, a carne, o café e trigo, entre outros diversos produtos (CLAUDINO et al., 2013). A cada ano, a participação brasileira no comércio internacional vem crescendo, com destaque para a produção de carne bovina, suína e de frango. Segundo o Ministério da Agricultura (MAPA), até 2020, a expectativa é que a produção nacional de carnes suprirá $44,5 \%$ do mercado mundial. O aumento na produção de alimentos cárneos é impulsionado pelo crescimento populacional em todas as esferas, tanto na mundial, nacional, quanto na estadual e municipal, pressionando os empreendimentos frigoríficos à aumentarem sua escala de produção, a fim de suprir a demanda de alimentos (COSTA, 2014).

Segundo Instituto Brasileiro de Geografia e Estatística (IBGE, 2013) o Rio Grande do Sul foi responsável por 11.250,00 mil toneladas de carne bovina e 138.619,00 mil toneladas de carne suína destinada ao mercado externo no ano de 2013. O Rio Grande do Sul ocupa posição de destaque nesse setor da agroindústria, possuindo 574 abatedouros de bovinos e 116 abatedouros de suínos licenciados pelo órgão estadual competente, a Fundação Estadual de Proteção Ambiental Henrique Luiz Roessler - RS, além dos abatedouros licenciados pelos órgãos ambientais municipais (FEPAM, 2014).

Paralelamente ao desenvolvimento acelerado do setor agroindustrial, para produção de alimentos cárneos, houve também uma maior geração de efluentes oriundos do seu processamento. Visto que as atividades de abate consomem um elevado volume de água, onde este, quase em sua totalidade tornase água residual. (TAVARES, 2013). A água residual, se não possuir tratamento de maneira adequada causam sérios problemas ambientais (BEUX et al., 2007). De acordo com Rabelo (2014), pode ser observado que os impactos ambientais gerados através dos abatedouros se concentram nas águas residuais de cada etapa do processo de abate. As águas residuais caracterizam-se por apresentar elevada concentração de matéria orgânica biodegradável, proveniente basicamente de proteínas e lipídios obtidos do animal sendo um efluente de difícil tratamento por atingir padrões altos de concentração de matéria orgânica e alto potencial poluidor.

No Rio Grande do Sul, os critérios para lançamento de efluentes em recursos hídricos superficiais foram estabelecidos pela Resolução CONSEMA n 128/2006, que concedeu prazo de 4 (quatro) anos para o atendimento integral dos padrões de emissão (RIO GRANDE DO SUL, 2006). Beux (2007) e Santos (2011) evidenciaram dificuldades no tratamento de águas residuais provenientes das atividades de abatedouros e frigoríficos, pois além destes efluentes estarem incorporados com uma elevada concentração de nitrogênio e fosforo, torna-se também difícil obter resultados satisfatórios com sistema de tratamento convencional.

Estudos realizados por Frick e Ferris (2011) diagnosticaram que de 11 (onze) frigoríficos avaliados no Estado do Rio Grande do Sul, somente 1 (um) atendia aos padrões de emissão estabelecidos pela legislação. Por conseguinte, dentre esses parâmetros, os que apresentavam maior dificuldade de adequação eram o nitrogênio e o fósforo.

Devido a importância desse setor no Rio Grande do Sul e considerando o elevado potencial poluidor dos efluentes gerados, o presente trabalho teve por objetivo avaliar as condições de lançamento de efluentes de 4 (quatro) plantas frigorificas, localizadas na Região de Pelotas/RS, em relação ao atendimento aos padrões de emissão NTK - nitrogênio total Kjeldhal, $\mathrm{N}^{-\mathrm{NH}_{3}}$ - nitrogênio amoniacal, $\mathrm{P}$ - fósforo total. 


\section{Materiais e Métodos}

\subsection{Seleção das agroindústrias}

Para a realização desse estudo foram selecionadas 4 (quatro) abatedouros de bovinos, suínos e ovinos, localizados na região de Pelotas/RS, os quais representam os empreendimentos de maior significância na cidade de Pelotas. As empresas foram identificadas da seguinte forma A, B, C e D. As empresas selecionadas possuem licença ambiental em vigor e estação de tratamento de efluentes compatível com a vazão e características do efluente a ser tratado. Os empreendimentos B, C e D possuem vazão de emissão de efluentes aproximada $50 \mathrm{~m}^{3} \cdot \mathrm{d}^{-1}$ e seus padrões de emissão de NTK e P são respetivamente $20 \mathrm{mg}$ N.L-1 e $4 \mathrm{mg}$ P.L-1. O empreendimento A possuiu vazão de $300 \mathrm{~m}^{3}$.d-1 e o padrão de emissão de NTK e P são $20 \mathrm{mg}$ N.L-1 para nitrogênio, e $3 \mathrm{mg}$ P.L-1 para o fósforo. As estações de tratamento existentes possuem estrutura semelhante e podem ser resumidas da seguinte forma: sistema de gradeamento, tanque de retenção de sólidos, peneira hidrodinâmica, lagoa anaeróbia, lagoa facultativa e lagoa com plantas emergentes.

\subsection{Avaliação dos laudos de analise}

Para a realização desse estudo foram selecionadas 4 (quatro) abatedouros de bovinos, suínos e ovinos, localizados na região de Pelotas/RS, os quais representam os empreendimentos de maior significância na cidade de Pelotas. As empresas foram identificadas da seguinte forma A, B, C e D. As empresas selecionadas.

\subsection{Analise estatística}

Os resultados obtidos foram avaliados utilizando Analytical Software STATISTIX 9 (2008). Para todas as análises o nível de significância adotado foi de 95\% ( $p<0,05)$. A análise de normalidade foi realizada pelo teste de SHAPIRO-WILK. A análise de variância foi realizada pelo TESTE DE TUKEY.

\section{Resultado da discussão}

Na Tabela 1 são apresentados os resultados médios obtidos no monitoramento de 2010, antes do término do prazo legal, e de 2014, após o término do prazo estabelecido pela Resolução CONSEMA n ${ }^{\circ}$ 128/2006, para o parâmetro NTK.

Tabela 1 - Resultados* de NTK em 2010 e em 2014

\begin{tabular}{|c|c|c|}
\hline \multirow{2}{*}{ Abatedouros } & 2010 & 2014 \\
\hline & NTK (mg.L $\left.{ }^{-1}\right)$ & NTK (mg. $\left.\mathrm{L}^{-1}\right)$ \\
\hline A & $99,3 \pm 29,3^{\mathrm{a} * *}$ & $29,6 \pm 6,2^{b}$ \\
\hline $\mathrm{B}$ & $86,0 \pm 11,4^{\mathrm{a}}$ & $20,0 \pm 1,1^{\mathrm{b}}$ \\
\hline $\mathrm{C}$ & $119,25 \pm 35,5^{\mathrm{a}}$ & $22,0 \pm 0,7^{\mathrm{b}}$ \\
\hline $\mathrm{D}$ & $28,6 \pm 13,4^{\mathrm{a}}$ & $34,5 \pm 17,8^{\mathrm{a}}$ \\
\hline adrão de emissão & 20,0 & 20,00 \\
\hline
\end{tabular}

*Resultados expressos em média \pm desvio padrão; ${ }^{* *}$ Letras diferentes entre linhas indicam diferença significativa por Tukey $(p<0,05)$ entre os períodos avaliados. 
Pode ser observado na Tabela 1 , que nenhum dos efluentes avaliados não atendeu aos padrões de emissão para NTK estabelecido durante o ano de 2010, com exceção do efluente da empresa D, considerando o desvio padrão registrado da empresa, que oscilou entre momento de atendimento com não atendimento. Já em 2014, pode-se observar que ocorreu redução significativa de NTK em comparação aos valores médios de 2010, porém, apesar dessa melhora nas condições de lançamento, somente a empresa B apresentou concentrações de NTK condizentes ao padrão de emissão estabelecido. Os resultados do monitoramento da empresa D são aqueles que apresentaram a maior variação, conforme pode ser verificado pelo desvio padrão. Assim como em 2010, o efluente dessa mesma empresa oscilou entre o atendimento e o não atendimento da legislação.

Na Tabela 2 são apresentados os resultados médios obtidos no monitoramento de 2010 e de 2014 para o fósforo total.

Tabela 2 - Resultados* de Fósforo em 2010 e em 2014

\begin{tabular}{ccc}
\hline \multirow{2}{*}{ Abatedouros } & 2010 & 2014 \\
\cline { 2 - 3 } & $\mathrm{P}\left(\mathrm{mg} \cdot \mathrm{L}^{-1}\right)$ & $\mathrm{P}\left(\mathrm{mg} \cdot \mathrm{L}^{-1}\right)$ \\
\hline A & $20,9 \pm 8,7^{\mathrm{a} * *}$ & $3,4 \pm 1,1^{\mathrm{b}}$ \\
B & $25,2 \pm 21,7^{\mathrm{a}}$ & $3,7 \pm 0,5^{\mathrm{b}}$ \\
C & $35,0 \pm 7,3^{\mathrm{a}}$ & $3,2 \pm 0,7^{\mathrm{b}}$ \\
D & $8,1 \pm 1,4^{\mathrm{a}}$ & $2,3 \pm 1,1^{\mathrm{b}}$ \\
\hline
\end{tabular}
Padrão de emissão
4,0
$4,0(3,0) * * *$

* Resultados expressos em média \pm desvio padrão; ${ }^{* *}$ Letras diferentes entre linhas indicam diferença significativa por Tukey $(\mathrm{p}<0,05)$ entre os anos avaliados; ${ }^{* * *}$ Até janeiro/13 o abatedouro A tinha padrão de emissão de $\mathrm{P} 4,0$ mg.L ${ }^{-1}$, como os demais. Após, o padrão de emissão passou para 3 mg. $L^{-1}$

Conforme observado na Tabela 2, em 2010 nenhum dos empreendimentos atendeu ao padrão de emissão de fósforo. Porem na avaliação em 2014 os empreendimentos B, C e D apresentaram resultados inferiores a $4 \mathrm{mgP} . \mathrm{L}^{-1}$, assim atendendo ao padrão de emissão. Também pode-se observar que houve redução significativa nos resultados de 2014 em comparação a 2010, o que indica que as adequações promovidas apresentaram resultados satisfatórios. A Empresa A, apesar desses resultados satisfatórios, ainda oscila entre o atendimento e o não atendimento ao limite de 3,0 mg P.L-1.

Cabe salientar que a Resolução CONSEMA 128/2006 ainda prevê o atendimento aos padrões estabelecidos por carga orgânica e por eficiência mínima de remoção, que para NTK, porém essa remoção deve ser analisada em conjunto com limite máximo de $\mathrm{N}-\mathrm{NH}_{3}$ de $20 \mathrm{mgN} . \mathrm{L}^{-1}$.

Na Tabela 3 é apresentada a eficiência média de remoção de NTK e P em 2014 e a concentração média de $\mathrm{N}-\mathrm{NH}_{3}$ no mesmo período.

Tabela 3 - Eficiência média de remoção de NTK e Fósforo em 2014

\begin{tabular}{cccc}
\hline Abatedouros & \multicolumn{2}{c}{ Eficiência de remoção (\%) } & $\begin{array}{c}\text { N-NH } \\
\left(m g . N . L^{-1}\right)\end{array}$ \\
\cline { 2 - 4 } A & NTK & P & $18,6 \pm 5,0^{*}$ \\
B & 54,5 & 49,4 & $15,0 \pm 1,1$ \\
C & 66,85 & 50,26 & $14,0 \pm 1,4$ \\
D & 79,63 & 63,22 & $24,0 \pm 12,1$ \\
\hline Padrão & 43,72 & 77,3 & 20,00
\end{tabular}

${ }^{*}$ Resultados expressos em média \pm desvio padrão 
Conforme pode ser observado, em 2014 somente a empresa $C$ atingiu a eficiência de remoção média de $79,6 \%$, acima do limite mínimo de $75 \%$ exigido pela legislação, conjuntamente com a concentração inferior a $20 \mathrm{mg} \cdot \mathrm{L}^{-1}$ enquadrando-se nos padrões de emissão da legislação. $\mathrm{O}$ empreendimento $\mathrm{D}$ também alcançou valor superior a $75 \%$ de remoção do fósforo, atendendo a legislação tanto em concentração como em eficiência mínima de remoção.

Durante o período analisado, as empresas investiram em boas práticas ambientais, como melhorias no sistema de gestão de resíduos, treinamento de pessoal, sistemas de gradeamento interno e redução de desperdício. Além disso, foram promovidas melhorias no sistema de tratamento, o que promoveu a redução significativa nas concentrações de $\mathrm{NTK}, \mathrm{N}-\mathrm{NH}_{3}$ e $\mathrm{P}$, com exceção da Empresa $\mathrm{D}$ em relação as formas de nitrogênio.

A redução na concentração de NTK ao longo do tempo pode ser visualizada na Figura 1

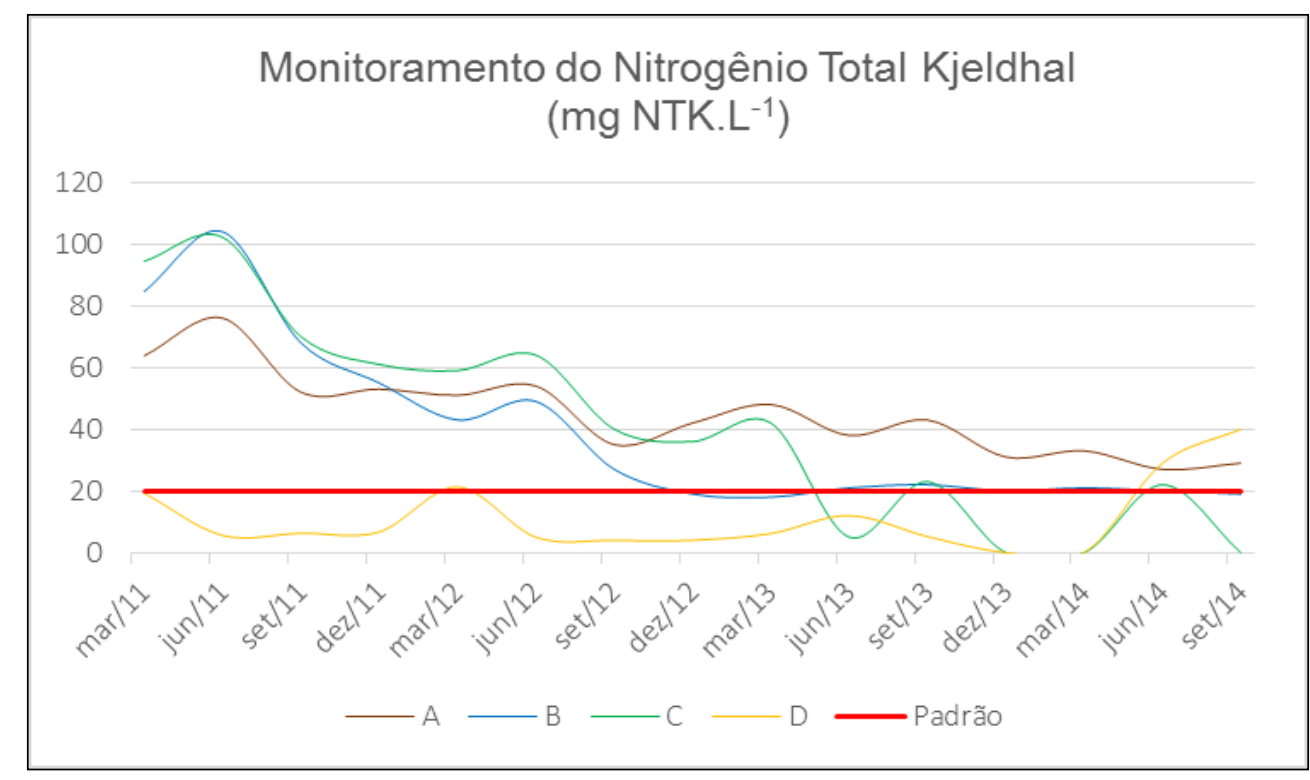

Figura 1 - Monitoramento do Nitrogênio Total Kjeldhal

Conforme pode ser observado, o NTK no efluente final dos empreendimentos A, B e C, apresentou redução de valor a partir do segundo trimestre/2011, enquanto que o efluente tratado da D manteve-se abaixo do limite estabelecido em praticamente todo o período avaliado. Pode se observar ainda que a empresa B começou a atender ao padrão de emissão a partir do quarto trimestre/2012, apesar de algumas flutuações de valores, enquanto que a $C$ passou a atingir valores menores que $20 \mathrm{mg} \mathrm{N} \cdot \mathrm{L}^{-1} \mathrm{a}$ partir de junho/13. Na Figura 2 são apresentados os resultados do monitoramento do nitrogênio amoniacal dos empreendimentos A, B, C e D em todo período avaliado.

Com base na Figura 2, pode-se concluir que as empresas A, B e C apresentaram redução no valor de concentração de nitrogênio amoniacal, a partir do mês de junho/2011, alcançando ao padrão de emissão após o segundo trimestre/2013. Já empreendimento D atendia ao padrão de nitrogênio amoniacal desde o início do monitoramento, porém houve uma pequena ultrapassagem em junho/2014. Diante da dificuldade de atender ao padrão de emissão de NTK a empresa D optou em promover a aplicação do efluente tratado no solo para fins agrícolas. Dessa forma, não lançando mais o efluente tratado em recursos hídrico superficial, essa empresa está atendendo integralmente ao previsto na legislação ambiental. 


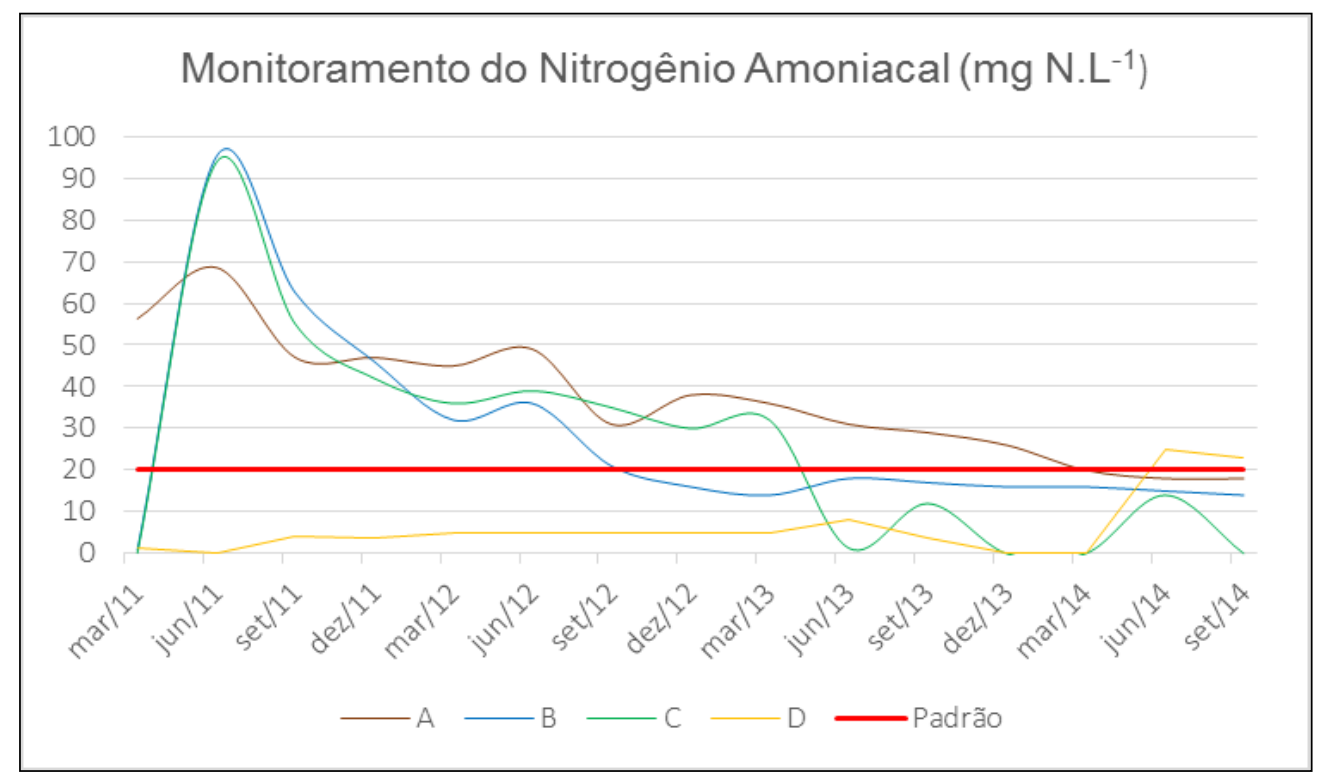

Figura 2 - Monitoramento do nitrogênio amoniacal

Na Figura 3 são apresentados os resultados do monitoramento de fósforo das empresas B, C e D, que possuem padrão de emissão de fósforo de $4 \mathrm{mg} \cdot \mathrm{L}^{-1}$.

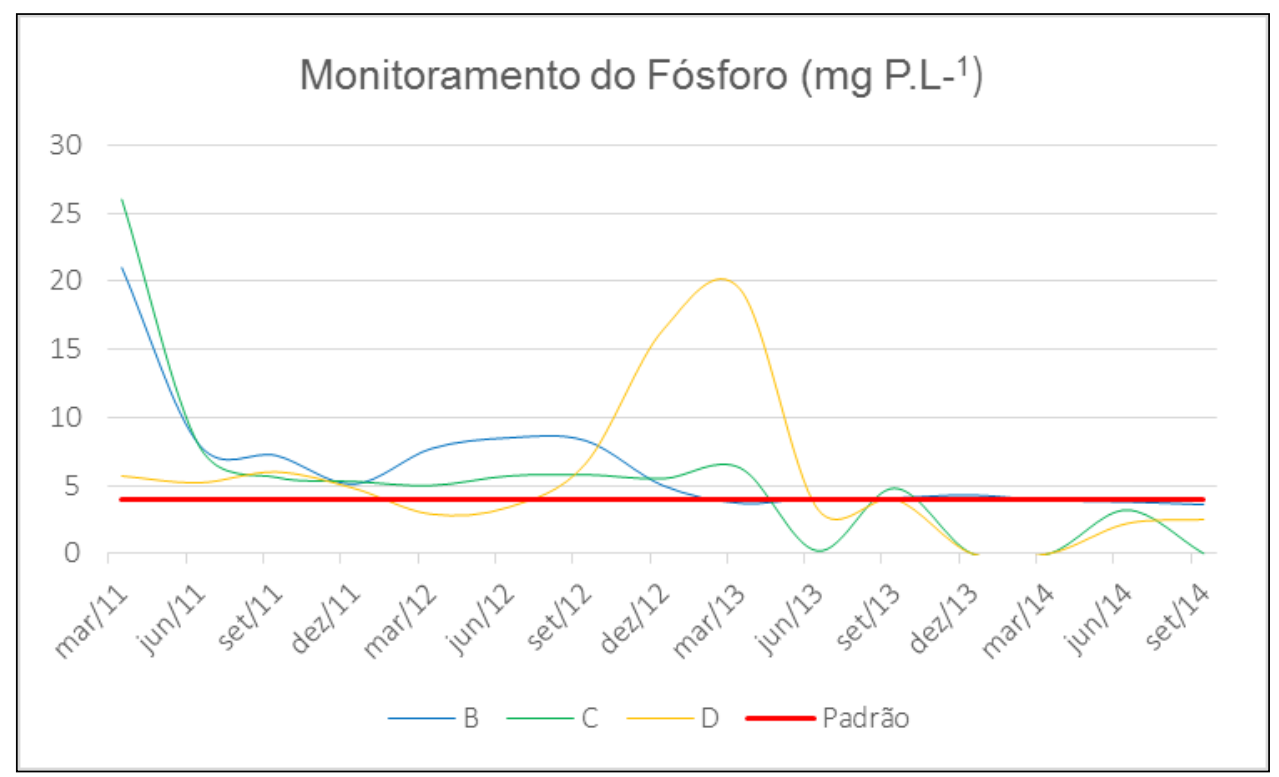

Figura 3 - Monitoramento do fósforo - empresas B, C e D

No segundo trimestre/2011 a concentração de fósforo apresentou redução em relação ao trimestre anterior para todas essas empresas, mantendo-se próximo ao padrão de emissão até junho/2013, quando o padrão de emissão começou a ser atendido. Atualmente, todas atendem ao limite de 4 mgP.L $\mathrm{L}^{-1}$. Pode se observar ainda que na empresa D ocorreu um aumento na concentração de fósforo entre dezembro/2012 e março/2013, período em que parte da estação ficou fora de operação devido às obras de adequação realizadas.

O comportamento do fósforo na empresa A, cujo padrão de emissão é $3 \mathrm{mg} \cdot \mathrm{L}^{-1}$, é apresentado na Figura 4. 


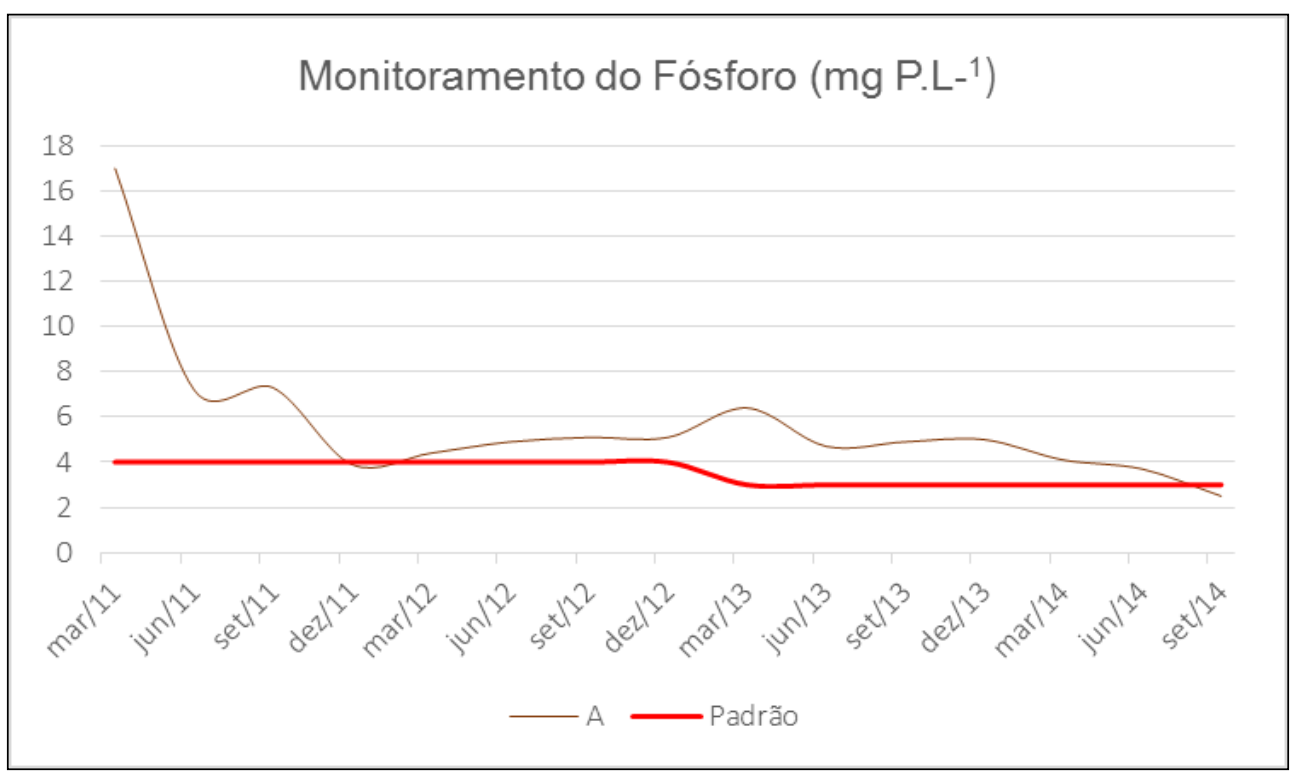

Figura 4 - Monitoramento do fósforo - empresa A

Das empresas avaliadas a empresa A possui a maior vazão licenciada $\left(300 \mathrm{~m}^{3} . \mathrm{d}^{-1}\right)$ justificando o padrão de emissão de fósforo diferente das demais empresas, que não ultrapassam $100 \mathrm{~m}^{3} \cdot \mathrm{d}^{-1}$.

No efluente da empresa A, assim como observado no efluente das outras empresas, a concentração de fósforo no efluente tratado também apresentou redução de valor no segundo trimestre/2011 em relação primeiro trimestre do mesmo ano. O padrão de emissão de $3 \mathrm{mg}^{-L^{-1}}$ somente começou a ser atingido em setembro/2014, porém, se o padrão fosse igual ao das empresas anteriores, já estaria sendo atendido desde junho/2014.

\section{Conclusão}

Todas as empresas avaliadas atendem atualmente ao padrão de lançamento estabelecido para fósforo. Em relação ao parâmetro NTK, 50\% das empresas avaliadas atenderam ao preconizado na legislação, por concentração ou por eficiência mínima de remoção seguida da concentração mínima de $\mathrm{N}-\mathrm{NH} 3$. Apesar das melhorias implantadas em todas as estações de tratamento de efluentes avaliadas, 2 (duas) empresas ainda necessitam promover novas adequações visando ao atendimento integral da resolução COSEMA n 128/2006.

\section{Referências}

APHA, Awwa. Wef. 2005. Standard Methods for the Examination of Water and Wastewater. 21st ed. American Public Health Association. Washington, DC. Part, v. 8000, p. 94-100.

BEUX, Simone; NUNES, Ezequiel; BARANA, Ana Cláudia. Effect of temperature on two-phase anaerobic reactors treating slaughterhouse wastewater. Brazilian Archives of Biology and Technology, v. 50, n. 6, p. 1061-1072, 2007.

BRASIL, Ministério da Agricultura, Pecuária e Abastecimento - MAPA. Disponível em:< http://www.agricultura.gov.br/ >. Acesso em: nov. 2014. 
Centro de Estudos Avançados em Economia Aplicada - CEPEA. Disponível em: < http://cepea.esalq.usp.br/ >. Acesso em: set. 2014.

CLAUDINO, Edison S.; TALAMINI, Edson. Análise do Ciclo de Vida (ACV) aplicada ao agronegócioUma revisão de literatura. R. Bras. Eng. Agríc. Ambiental, v. 17, n. 1, p. 77-85, 2013. Disponível em: < http://www.scielo.br/pdf/rbeaa/v17n1/v17n01a11.pdf >. Acesso em: nov. 2014.

COSTA, Rodrigo Fagundes et al. Valores econômicos estimados para objetivos em sistemas de produção de ciclo completo no sul do Brasil. In: Embrapa Pecuária Sul-Artigo em anais de congresso (ALICE). In: Seminário Interinstitucional de Ensino, Pesquisa e Extensão, 18.; Mostra de Iniciação Cientifica, 16,; Mostra de Extensão, 11., 2013, Cruz Alta. Ciência, conhecimento e sociedade de risco: anais. Cruz Alta: Unicruz, 2013., 2014. Disponível em: < http://www.alice.cnptia.embrapa.br/bitstream/doc/979287/1/Cardosovalores.pdf >. Acesso em: nov. 2014.

Fundação Estadual de Proteção Ambiental Henrique Luiz Roessler - RS - FEPAM. Disponível em: < http://www.fepam.rs.gov.br/ >. Acesso em: dez. 2014.

FRICK, J. \& Ferris, L. Estudo do monitoramento de efluentes líquidos industriais de frigoríficos no Rio Grande do Sul. TCC - Trabalho de Conclusão de Curso de Engenharia Química - UFRGS. 2011. Disponível em < http://www.lume.ufrgs.br/handle/10183/36896 >. Acessado em: nov. 2014.

Instituto Brasileiro De Geografia e Estatística - IBGE. Indicadores 2013: Estatística da produção pecuária - primeiro trimestre 2013. Disponível em: < http://www.ibge.gov.br/home/estatistica/indicadores/agropecuaria/producaoagropecuaria/abateleite-couro-ovos_201301_publ_completa.pdf >. Acesso em: dez. 2014.

Instituto Brasileiro De Geografia e Estatística - IBGE. Indicadores 2013: Estatística da produção pecuária - segundo trimestre 2013. Disponível em: < http://www.ibge.gov.br/home/estatistica/indicadores/agropecuaria/producaoagropecuaria/abateleite-couro-ovos_201302_publ_completa.pdf >. Acesso em: dez. 2014.

Instituto Brasileiro De Geografia e Estatística - IBGE. Indicadores 2013: Estatística da produção pecuária - terceiro trimestre 2013. Disponível em: < http://www.ibge.gov.br/home/estatistica/indicadores/agropecuaria/producaoagropecuaria/abateleite-couro-ovos_201303_publ_completa.pdf >. Acesso em: dez. 2014.

Instituto Brasileiro De Geografia e Estatística - IBGE. Indicadores 2013: Estatística da produção pecuária - quarto trimestre 2013. Disponível em: < http://www.ibge.gov.br/home/estatistica/indicadores/agropecuaria/producaoagropecuaria/abateleite-couro-ovos_201304_publ_completa.pdf >. Acesso em: dez. 2014. 
JAIME, Patrícia Constante et al. Ações de alimentação e nutrição na atenção básica: a experiência de organização no Governo Brasileiro. Rev. nutr, v. 24, n. 6, p. 809-824, 2011. Disponível em: < http://www.scielo.br/pdf/rn/v24n6/01v24n6.pdf >. Acesso em: dez. 2014.

RABELO, Mariane Helena Sances; SILVA, Eric Keven; PERES, Alexandre de Paula. Failure Mode and Effect Analysis for the evaluation of environmental impacts of animal slaughter. Engenharia Sanitaria e Ambiental, v. 19, n. 1, p. 79-86, 2014. Disponível em: < http://www.scielo.br/pdf/esa/v19n1/1413-4152-esa-19-01-00079.pdf >. Acesso em: nov. 2014.

RIO GRANDE DO SUL, Fundação Estadual de Proteção Ambiental FEPAM -RS. Disponível em:< http://www.fepam.rs.gov.br/ >. Acesso em: nov. 2014.

RIO GRANDE DO SUL, 2006 - Secretaria do Meio Ambiente - Conselho Estadual do Meio Ambiente Resolução CONSEMA № 128, de 24 de novembro de 2006. Dispõe sobre a fixação de Padrões de Emissão de Efluentes Líquidos para fontes de emissão que lancem seus efluentes em águas superficiais no Estado do Rio Grande do Sul.

SANTOS, Wilson Roberto B dos. Remoção de nitrogênio e fósforo presentes em efluentes agroindustriais pela precipitação de estruvita. Dissertação de mestrado - UFSM. 2011. Disponível em: < http://cascavel.cpd.ufsm.br/tede/tde_busca/arquivo.php?codArquivo=4005 >. Acessado em dez. 2014

TAVARES, Adenir Paulo; CHRISTMANN, Jonatan André. Tratamento e reuso de água em abatedouro de suínos. TCC - Trabalho de Conclusão de Curso em Gestão Ambiental. UTFPR. 2014. Disponível em: http://repositorio.roca.utfpr.edu.br/jspui/bitstream/1/1661/1/MD_COGEA_2013_1_04.pdf >. Acesso em: nov. 2014.

TRICHES, Rozane Marcia; GERHARDT, Tatiana Engel; SCHNEIDER, Sergio. Políticas alimentares: interações entre saúde, consumo e produção de alimentos. Interações: Revista Internacional de Desenvolvimento Local. Campo Grande. Vol. 15, n. 1 (2014), p. 109-20, 2014. Disponível em: < http://www.scielo.br/pdf/inter/v15n1/v15n1a11.pdf >. Acesso em: nov. 2014. 